\title{
طريقة ترجمة الكتب العربية إلى الإنجليزية و الإندونيسية بمعهد فونجاء دار السلام الإسلامي فوتوءان دايا باميكاسن
}

\author{
Noer Fadila ${ }^{1}$ \\ 1(IAIN Madura/dilolottt@gmail.com) \\ Umar Bukhory ${ }^{2}$ \\ 2(IAIN Madura/bukhory06@gmail.com)
}

\begin{abstract}
There is a translation program of Arabic books into English and Indonesian with different methods applied in Pondok Pesantren Puncak Darussalam. This research is to describe translation methods of Arabic books into English or Indonesian and to analyze similarities and differences between the translation methods in two language centers. This is a descriptive qualitative research. It revealed that there are three methods in the translation of Arabic books. Semantic translation (with guidance) and free translation (without guidance) used in the translation of the Arabic books into English in English center, and word-for-word translation used in the translation of Arabic books into Indonesian at the Arabic center. Meanwhile, the similarities are they used the introductory translation; oral translation, word-for-word translation, and the translation process that is still oriented to the source language. The differences are the translation process at English center is more (used two methods) than in Arabic center (used one method), and the use of semantic translation methods in Arabic center inserted elements of semantics, while the application of word-forword translation in Arabic center inserted the elements of nahwu. Semantic translation in English center included in the category of understanding, meantime word-for-word translation included in the level of analysis.
\end{abstract}

\section{Keywords:}

Translation Method, Arabic Book, Language Center

DOI: $10.19105 /$ ojbs.v12i2.2017

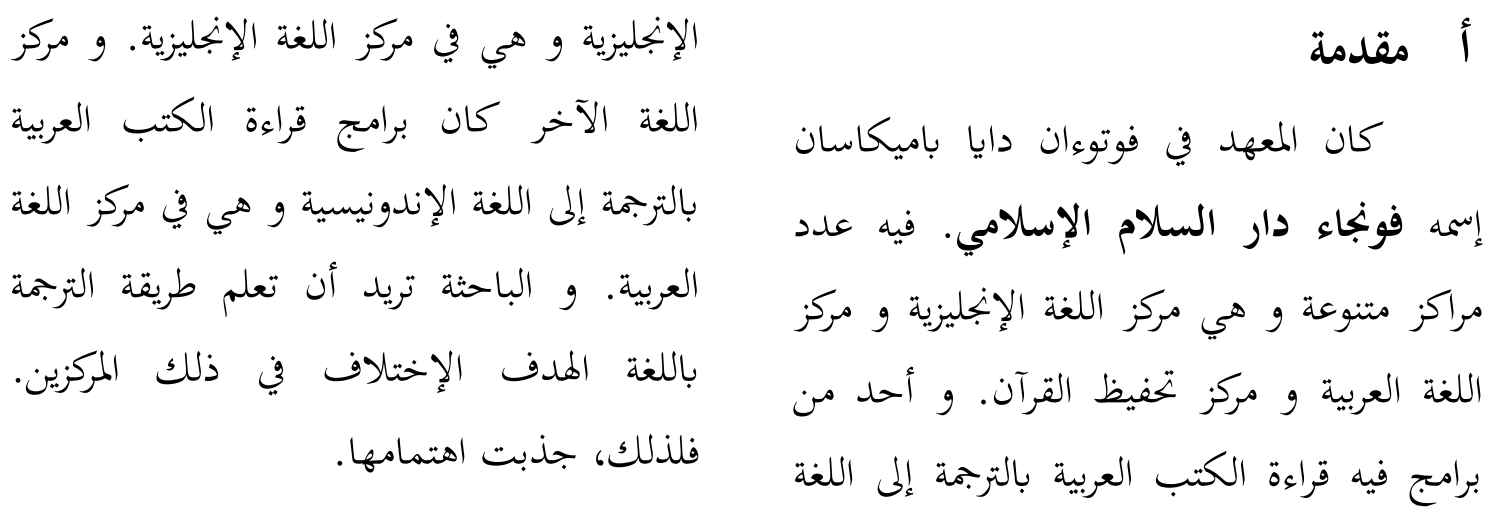


و على هذا البحوث القديمة لهذا البحث

عن طريقة الترجمة و هي Muhammad

Pembelajaran 2014، Fahaddudin

Kitab Kuning Melalui Metode

Tarjamah di Pondok Pesantren Al-

Munawwir Yogyakarta

عملية التعليم، تنفيذ طريقة تعليم الترجمة المستخدم

في مدرسة الدينية في معهد "المنور كومفليك نور

السلام" وهي طريقة "معنى غاندول" و طريقة

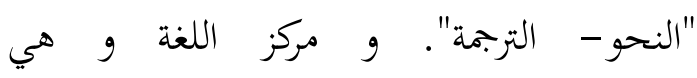

،Muhammad Hidayaturrahman

Peran 2015

Pengembangan Bahasa Asing dalam

Meningkatkan

Kemampuan

Berbicara Bahasa Arab Siswa (Studi Kasus di Madrasah Aliyah Jâ a alHaq Kota Bengkulu) اللغة (LPBA) ادوار و هي دور المخطط والمنفّذ لبرنامج النشاط في ارتفاع مهارة الكلام

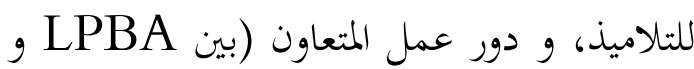
هي مدير MA "جاء الحق"، و مدير معهد "سينتوط عليباشا"، و جامع التلاميذ في "جاء الحق")، و دور التنسيق (بين المؤسسة بمدير

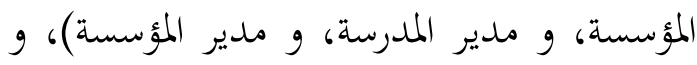
دور المقاربة في تنفيذ النشاط، و دور الحث (مثل مدافع يدفع التلاميذ ليتبع برامج النشاط في ارتفاع مهارة الكام باللغة العربية).

كان اختلاف الدراسة بين بحوث القديمة هذذا البحث. أمّا دراسة بحوث القديمة و هي
و الترجمة عملية تتحقق باللغات، أي أها

عملية استبدال نص في لغة معينة بنص آخر في

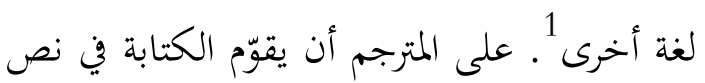

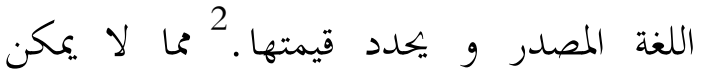
الإحاطة به الا بالمطالعة المستفيضة و النظرة

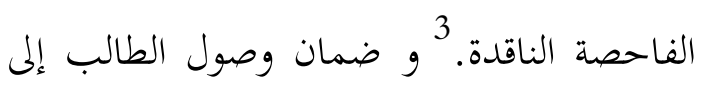
الاستجابة الصحيحة و بالتالي الانخفاض الشديد

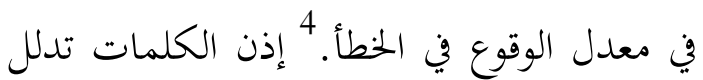
المترادفات هي الترجمة من ذلك المعنى. و طريقة الترجمة و هي كيفية التي يستعملها المترجم حين يقضى أن يترجم نص المنبع. نيومارك (911) (191) يقسم طريقة الترجمة

على فرقتين و هي ضغط إلى اللغة المنبع (BSu) و ضغط إلى اللغة الهدف.6 الدكتور رونالد ح. باتحغاتي قال عن سبعة عناصر من عملية الترجمة

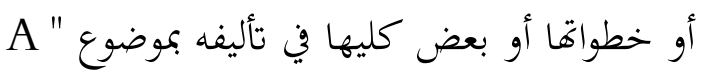
Survey of Translation Theory مطالعة و محلل و فهم و مصطلحي و عوامة و فحص و كلام. 7 1 عبد الباقي الصافي، نظرية لغوية للترجمة، (البصرة : دار الكتب، (19人

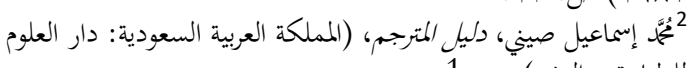

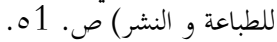

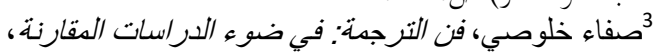

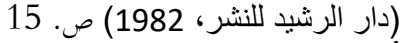

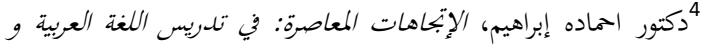

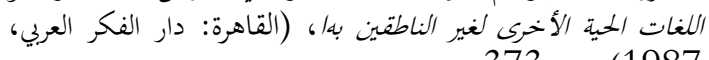
1987 ص. 373

${ }^{5}$ Moch. Syarif Hidayatullah, Jembatan Kata: Seluk-Beluk Penejemahan Arab-Indonesia, (Jakarta: Grasindo, 2017), 36.

${ }^{6}$ Rochayah Machali, Pedoman Bagi Penerjemah, (Bandung: PT Mizan Pustaka, 2009), 76.

7 A. Widyamartaya, Seni Menerjemahkan, (Yogyakarta: Anggota IKAPI, 1989), 15. 


\section{ب منهج البحث}

تستخدم الباحثة المنهج النوعي يعنى أن الن متحن

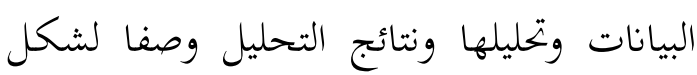

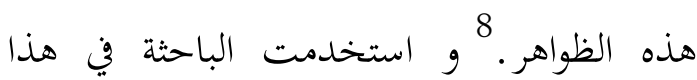

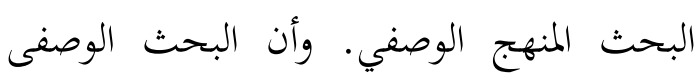

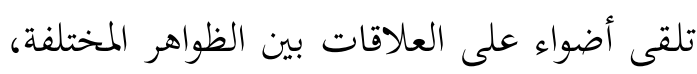

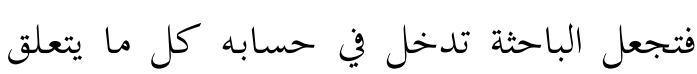
بالمشكلة. 9 وسوف يبدأ هذا البحث العلمى لمدة

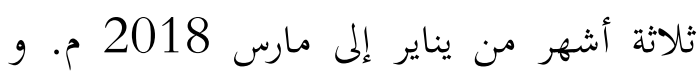

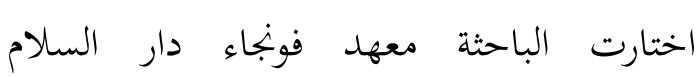

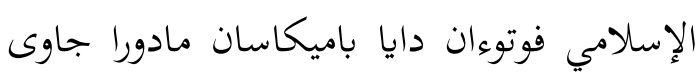
الشرقية و مسافة إلى عاصمة المحافظة حوالي 12

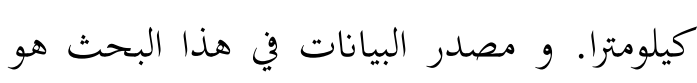
مدرّس الكتب العربية و الطلاب في مركز اللغة الإنجليزية و مركز اللغة العربية فونجاء دار السلام

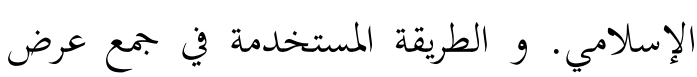

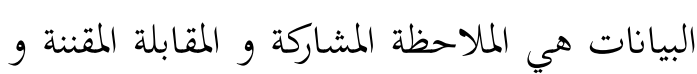

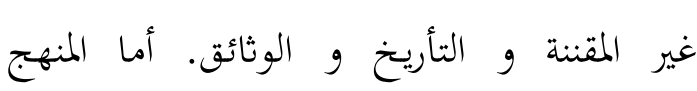
المستخدم في تحليل البيانات هو منهج الدراسة الونات الوصفية.

و الملاحظة هي وسيلة يستخدمها الإنسان

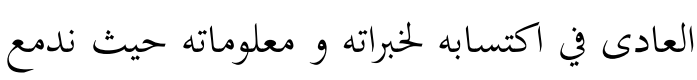

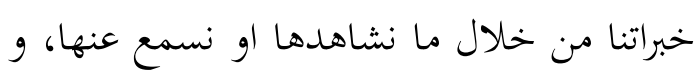
لكن الباحثة حين يلاحظة فإنه تتبع منهجا معينا

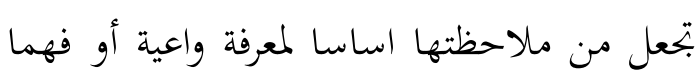

${ }^{8}$ Lexy J. Moleong. Penelitian Kualitatif (Bandung: Remaja Rosdakarya, 2011).

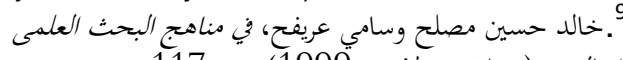

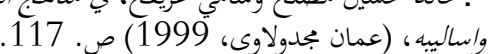

الدراسة عن استخدام نوع طريقة الترمة للكتب

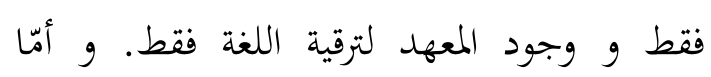
دراسة هذا البحث و هي الدراسة عن طريقة لكنة الترجمة الكتب العربية إلى اللغة الإنجليزية في مركز

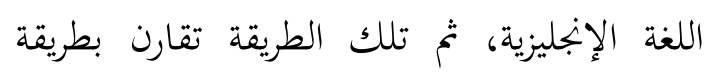
التربمة الكتب العربية إلى اللغة الإندونيسية في العيدة مركز اللغة العربية. و على هذا، تمدف الباحثة إلى أربعة

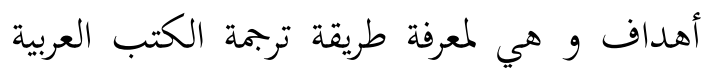
إلى اللغة الإنجليزية فيمركز اللغة الإنجليزية بعهدا

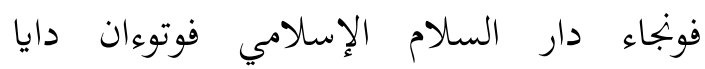
باميكاسن، و لمعرفةطريقة ترجمة الكتب العربية إلى الى الكائ اللغة الإندونيسية في مركز اللغة العربيةكعهد فونجاء العيكاء

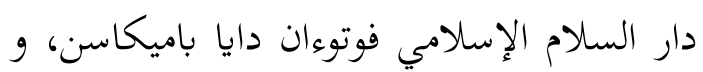
لمعرفة أوجه التشابه بين طريقة ترجمة الكتب العربية

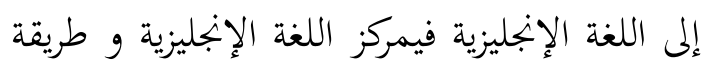
ترجمة الكتب العربية إلى اللغة الإندونيسية في مركز

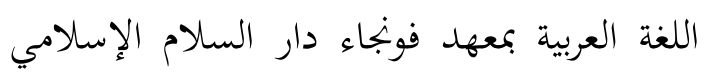

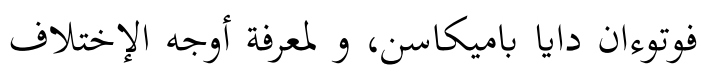
بين طريقة ترجمة الكتب العربية إلى اللغة الإنجليزية

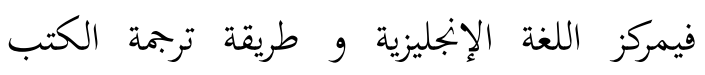
العربية إلى اللغة الإندونيسية في مركز اللغة العربية بعمهد فونجاء دار السلام الإسلامي فوتوعان دايا

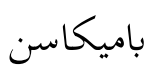


الرابع، المرشد يترجم النص المقروء إلى اللغة

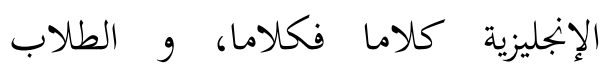
يستمعون قرائته ثم يكتبون الترجمة.

الخامس، المرشديسأل الطلابليقرؤوا الكتاب بترجمة اللغة الإنجليزية كما يقرأها المرشلـ.

الساد،،المرشد يعطي الوقت إلى الطلاب

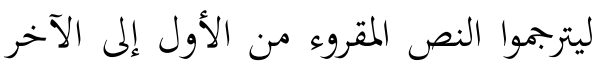
إلى اللغةالإنجليزية بلغتهم.

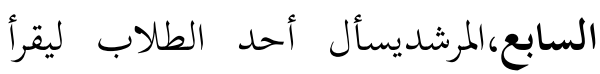
نتيجته و يبينها إلى أصدقائه. 12

عملية التربمة بالإرشاد و هي عملية الترجمة من الرابع حتى الخامس التي يرشد

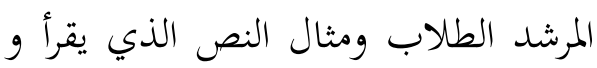
يترجم إلى اللغة الإنجليزية في مركز اللغة الإنجليزية كما يلي:

"دليل المالكية: و استدل المالكية" على أن البسملة ليست آية من الفاتحة، ولا من القرآن و إنما هي التبرك." (تفسير آية الأحكام: 38 (38) فترجمتهاكما يلي:

\begin{tabular}{|c|c|}
\hline $\begin{array}{l}\text { The postulate of } \\
\text { Malikiyah }\end{array}$ & دليل المالكية \\
\hline $\begin{array}{l}\text { Malikiyah infer that } \\
\text { Basmalah is not verse } \\
\text { of Fatihah and is not } \\
\text { post of Al-Qur'an }\end{array}$ & و لعلى أن البسملة المالكية \\
\hline
\end{tabular}

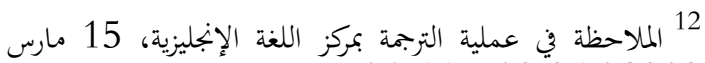

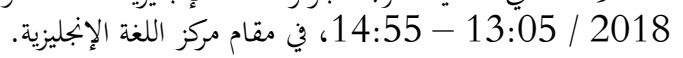

دقيق لظاهرة معينة . 10 وهناك نوعان من

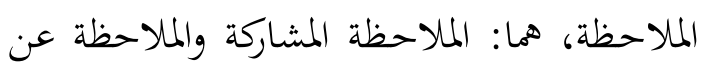

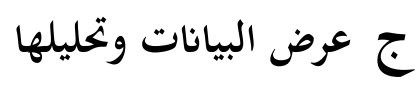
1.طريقة ترجمة الكتب العربية إلى اللغة الإنجليزية في مركز اللغة الإنجليزية فونجاء دار السلام الإسلامي فوتوءان دايا

$$
\text { باميكاسن }
$$

برنامج ترجمة إلى اللغة الإنجليزية عمليتان و هي عملية التربمة بالإرشاد وعملية الترجمة بدون الإرشاد. و لكلعملية الترجمة الطريقة المختلفة ولو ترجمة الكتب العربية إلى اللغة الإنجليزية متشابها. عملية التربمة في مركز اللغة الإنجليزية كما يلي: أولا، المرشد يقرأ الكتاب و معناها باللغة الإندونيسية بكلمة فكلمة، و الطلاب يستمعون و يكتبون بعض المعنى التي

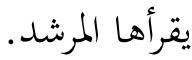
الثاني، المرشد يبين ما يقرأها إلى الطلاب.

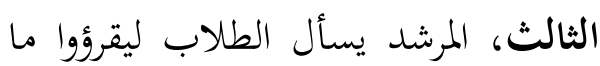

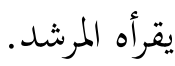


و قال عبد الرحمن المرشد في مركز اللغة الإنجليزية:

"استعمال تلك الطريقة لتصير الهوية في مركز اللغة الإنجليزية. و هي التييخالف مركز اللغة الإنجليزية بخارجه. أي تستعمل هذه الطريقة للصوري في قراءة

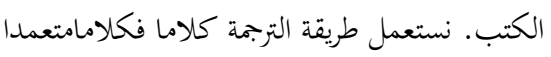
و نحن لا ندرس عن صيغة الكلمة و ترتيب الكلام في الترجمة إلى اللغة الإنجليزية. إذن، نغتار الكلام

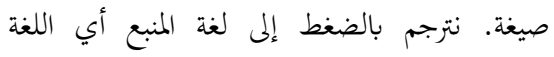
العربية."13

من ناحية عملية الترجمة و مثال النص "تفسير آية الأحكام: ص. 38" و نتيجة الترجمة "تفسير آية الأحكام: ص. 3.8" و قول عبد الرحمن المرشد في مركز اللغة الإنجليزية استعمال طريقة الترجمة بكالاما فكلاما في مركز اللغة الإنجليزية. و قول السيد عبد الرحمن"في هذه الترجمة كلاما فكلاما نطلب حق المعنى من تلك نص اللغة العربية، و لكن تركيب كلمتها تتبع على نظام اللغة الإنجليزية."14 هذه الطريقة موافق بطريقة الترجمة التي تكتب زاكا الفريسي عن طريقة الترجمة الدلالية، "هذه الترجمة يركز بحث النظير في نحو الكلمة، و لكنها مربوط بثقافة لغة إلى اللغة المترجمة منها. ولو كذلك، تسعى الترجمة أن تحوّل معنى الموضوعيّ من لغة المنبع بتركيب علم النحوي و تركيب

\begin{tabular}{|l|r|}
\hline & $\begin{array}{r}\text { الفاتحة، ولا من } \\
\text { و إنما هي التبرك } \\
\hline \begin{array}{l}\text { But, this is to get } \\
\text { blessing }\end{array}\end{array}$ \\
\hline
\end{tabular}

و الباحثة تسأل طالبين ليترجما أحد

الكتب اللغة العربية في مركز اللغة

الإنجليزية. و هما يترجمان كتابتفسير آية

الأحكام : ص. 308

و هذا نتيجة الترجمة من الطلاب

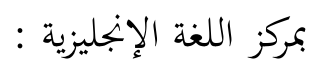

\begin{tabular}{|c|c|}
\hline The religious low & الأحكام الشرعية \\
\hline The first low & الحكم الأول \\
\hline $\begin{array}{l}\text { Is hijab obligated } \\
\text { for all of womens? }\end{array}$ & هل هميع النباء الحجاب على \\
\hline $\begin{array}{l}\text { The verse } A l- \\
\text { Karimah indicate }\end{array}$ & يدل ظاهر الأية الكريمة \\
\hline $\begin{array}{l}\text { That hijab is } \\
\text { obligated to all of } \\
\text { mukallaf moslem } \\
\text { women by syara' }\end{array}$ & على على أن الحجاب مفروض المؤمنات \\
\hline And they are & و هن \\
\hline Muslims & المسلمات \\
\hline Independent & الحرائر \\
\hline Mature women & البالغات \\
\hline As Allah decree & لقوله تعالى \\
\hline $\begin{array}{l}\text { Oh Prophet, tell to } \\
\text { your wives and } \\
\text { your } \\
\text { daughtersand the } \\
\text { women of } \\
\text { believers: to draw } \\
\text { their veil all of } \\
\text { over their body }\end{array}$ & 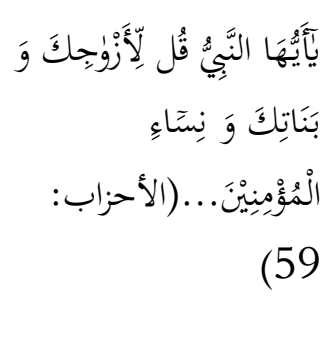 \\
\hline
\end{tabular}

\footnotetext{
13 صباحا في المصلى فونجاء دار السلام. بعابد

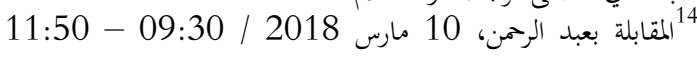
صباحا في المصلى فونجاء دار السلام.
} 
Challenge Theory ) النظرية و التطبيق and Practice التحدى فيما قبل، و ذكرنا أن الجامعات التطبيقية حاولت أن توجد صيغة للتوازن بين الجانب النظري و التطبيقي في برامج الترجمة لاسيما و أن كثيرًا من هذه الجامعات قد أدركت أن التطور النظري لا يعنى بالضرورة تطورًا في المجالات التطبيقية أوو العكس، و ذلك لأهمية العنصر (البيداجوجي) في برامج الترجمة."18

تلك الطريقة موافق بطريقة الترجمة

التي تكتب زاكا الفريسي عن طريقة الترجمة الحرية، "هذه الترجمة تحاصل المادة الخاصة بدون كيفية خاصة. في هذا الحال، مترجم يحاصل الملى فقط بدون أن يهتم بالصغة."19 و "حين يترجم المترجم بهذه الطريقة، المترجم يفضل الملى و يضحى الصغة من نص لغة المنبع."

فعملية الترجمة بدون الإرشاداستعمال

طريقة الترجمةالحريةتعنيأخذ النقطة المهمة في الموضوع ثم يترجمون (أي الطلاب في مركز اللغة الإنجليزية) إلى اللغة الإنجليزية حرية بتلك النقطة المهمة.
الدلالي من لغة الهدف."15 و "حين يترجم المترجم بهذه الطريقة، المترجم لائق كثير و لدن كثير من المترجم يستعمل الترجمة الوفية." ل16

فعملية الترجمة بالإرشاداستعمال طريقة الترجمالدلالية تعنيأن يتماثل المترجم (أي المرشد في مركز اللغة الإنجليزية) بلغة المنبع (أي اللغة العربية) في تطبيق استعمال هذه الطريقة و لكن تركيب كلمتها تتبع على نظام اللغة الإنجليزية. و عملية الترجمة بدون الإرشادو هي عملية الترجمة من الرابع حتى الخامسالتي يترجمون الطلاب النص المقروء بنفسه. قال السيد عبد الرحمن علي بحرين: "أمّا طريقة Main Mapping الترجمة تستعمل الطريقة و هي أخذ النقطة المهمة في الموضوع ثم نأمر الطلاب لأن يترجمون إلى اللغة الإنجليزية حرية بتلك النقطة المهمة."17

من ناحية عرض البيانات بمركز اللغة Main الإنجليزية عن هذه طريقة الترجمة Mapping لأنالبروفسور يوسف نور عوضقال في كتابه "علم النص و نظرية الترجمة" عن تحدى

15 Mohammad Zaka Al-Farisi. Pedoman Penerjemahan: Arab (Bandung: PT Remaja Rosdakarya, 2011), 55.

16 Moch.Syarif Hidayatullah, Jembatan Kata: Seluk-Beluk Penejemahan Arab-Indonesia. 40. 17 المقابلة بعبد الرحمن، 10 مارس 10 صباحا في المصلى فونجاء دار السلام.

${ }_{20}^{9}$ Al-Farisi. Pedoman Penerjemahan: Arab. 55

20 Moch.Syarif Hidayatullah, Jembatan Kata: Seluk-Beluk Penejemahan Arab-Indonesia. 41. 


\begin{tabular}{|c|c|}
\hline Adapun Kalimat & الكالام \\
\hline Yaitu adapun kalimat & هو \\
\hline Yaitu lafadz & اللفظ الم \\
\hline Yang tersusun & المركب \\
\hline Yang memberi faidah & المفيد \\
\hline $\begin{array}{l}\text { Dengan menggunakan bahasa } \\
\text { Arab }\end{array}$ & بالوضع \\
\hline
\end{tabular}

و الباحثة تسأل طالبين ليترجما أحد

الكتب اللغة العربية في مركز اللغة العربية،

و هي كتابتفسير آية الأحكام : ص.

308

و هذا نتيجة الترجمة من الطلاب

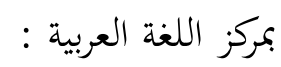

\begin{tabular}{|c|c|}
\hline $\begin{array}{l}\text { Adapun ini adalah hukum- } \\
\text { hukum }\end{array}$ & الأحكام \\
\hline Yang bersifat syari'ah & الشرعية \\
\hline Hukum yang pertama & الحكم الأول \\
\hline Apakah wajib & هل يجب \\
\hline Apa hijab & الحجاب \\
\hline Atas seluruh perempuan & على جميع \\
\hline Menunjukkan & يدل \\
\hline Apa zhahir ayat & ظاهر الأية \\
\hline Yang mulia & الكريمة \\
\hline Bahwa sesungguhnya hijab & على أن الحجاب \\
\hline
\end{tabular}

إذن،طريقة الترجمة تستعمل في الترجمة

إلى اللغة الإنجليزية في مركز اللغة الإنجليزية فونجاء دار السلام الإسلامي فوتوءان دايا باميكاسنو هي ريقة الترجمةالدلالية بالإرشاد وطريقة الترجمةالحريةبدون

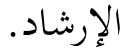

\section{2. طريقة ترجمة الكتب العربية إلى اللغة} الإندونيسية في مركز اللغة العربية فونجاء دار السلام الإسلامي فوتوءان دايا باميكاسن

برنامج ترجمة إلى اللغة الإنجليزية

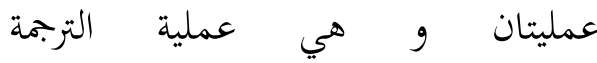
بالإرشادفقط. و عملية الترجمة في مركز

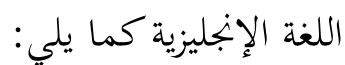
أولا، المرشد يقرأ الكتاب و معناها باللغة لئل الإندونيسية بكلمة فكلمة، و و الطلاب يستمعون و يكتبون بعض المعنى التي

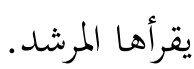
الثاني، المرشد يبين ما يقرأها إلى الطلاب. الثالث، المرشد يسأل الطلاب ليقرؤوا ما يقرأه المرشد. و مثال النص الذي يقرأ و يترجم إلى رهم اللغة الإندونيسية في مركز اللغة العربية كما يلي:"الكلام هو اللفظ المركب المفيد

21 الملاحظة في عملية الترجمة بمركز اللغة العربية، 15 مارس 2018 / 13:05 - 14:55، في مقام مركز اللغة الإنجليزية. 
من ناحية عملية الترجمة و مثال

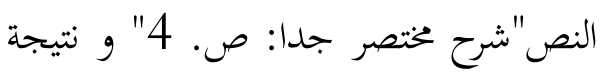

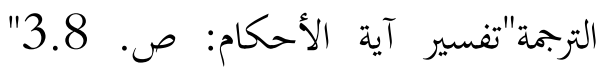

وقول السيد الترمذيلمرشد في مركز اللغة

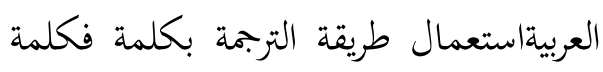
فيمركز اللغة العربية.و هدف استعمال هذه الطريقة و هي ليعلموا الطلاب المعنى من

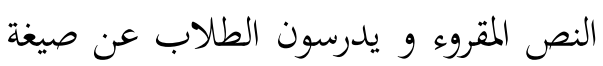
الكلمة و ترتيب الكلام من ذلك النص.

تلك طريقة الترجمة تكون في نظرية م.

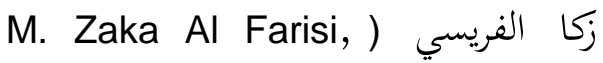
في كتابه قال "هذه الترجمة تصور (M.Hum بالترجمة صفا فصفا أي لغة الهدف تحت فال كلمات لغة المنبع. هذه الطريقة تركز كلمة

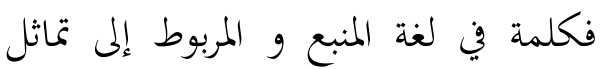

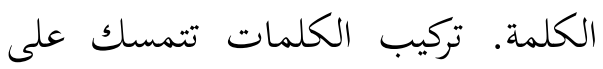
نص المنبع."23 و "حين يترجم المترجم هكذه تكلي الطريقة، فيضع الكلمات من نص الهدف في نص المنبع."24 المريقة، فيفع

إذن، طريقة الترجمة تستعمل في

الترجمة إلى اللغة الإندونيسية في مركز

اللغة العربية فونجاء دار السلام الإسلامي الإندونية في مركي

فوتوءان دايا باميكاسن و هي طريقة دار العام الإلاعي

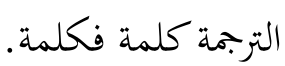

\begin{tabular}{|c|c|}
\hline Adalah difardlukan & مفروض \\
\hline $\begin{array}{l}\text { Atas seluruh perempuan } \\
\text { mukmin }\end{array}$ & على جميع \\
\hline Yang mukallaf-mukallaf & المكلفات \\
\hline Secara syara' & شرعا \\
\hline Adapun mereka & و هن \\
\hline $\begin{array}{l}\text { Adalah perempuan- } \\
\text { perempuan muslim }\end{array}$ & المسلمات \\
\hline Yang merdeka & الحرائر \\
\hline Yang baligh & البالغات \\
\hline $\begin{array}{l}\text { Berdasarkan firman Allah } \\
\text { Ta'ala }\end{array}$ & لقوله تعالى \\
\hline Wahai Nabi & يَآيَيُهَا النَّبُُّ \\
\hline $\begin{array}{l}\text { Mengatakanlah siapa } \\
\text { Engkau }\end{array}$ & قُلْ \\
\hline Kepada istri-istrimu & 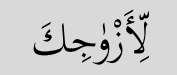 \\
\hline $\begin{array}{l}\text { Dan anak-anak } \\
\text { perempuanmu }\end{array}$ & وَ بَنَاتِكَكَ \\
\hline $\begin{array}{l}\text { Dan perempuan- } \\
\text { perempuan mukmin }\end{array}$ & 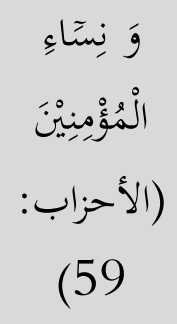 \\
\hline
\end{tabular}

و قال السيد الترمذيالمرشد في مركز

اللغة العربية: "أمّا طريقة الترجمة في مركز

اللغة العربية بطريقة الترجمة كلمة فكلمة

أيضا. و استعمال هذه الطريقة ليدرسوا

الطلاب عن صيغة الكلمة و ترتيب الكلام في علم اللغة العربية."22

${ }^{23}$ Al-Farisi, Pedoman Penerjemahan: Arab. 53.

${ }^{24}$ Moch. Syarif Hidayatullah, Jembatan Kata:

Seluk-Beluk Penejemahan Arab-Indonesia. 37.

1222 ملمقابلة بالسيد الترمذي، 10 مارس 2018 / 09:30 - 11:50 صباحا في المصلى فونجاء دار السلام. 


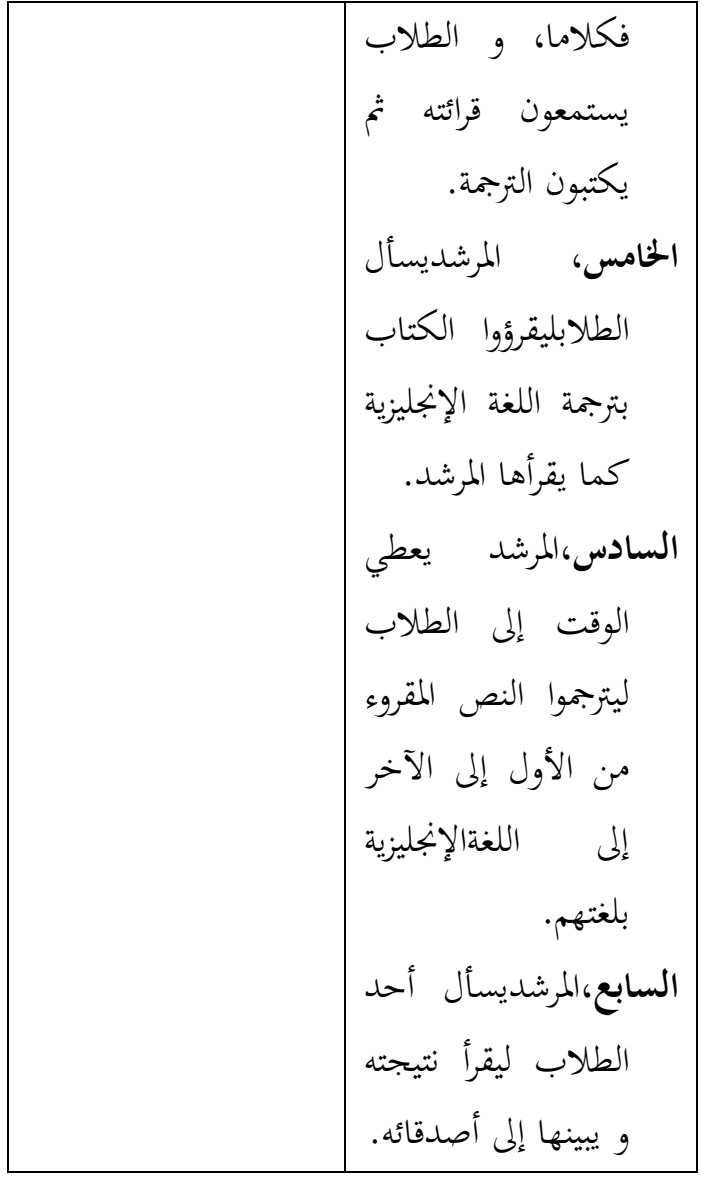

في تلك عملية الترجمة أوجه

التشابه و هي وجوه الإرشاد في مركز اللغة الإنجليزيةو في مركز اللغة العربية. أما وجوه الإرشاد فيسمى الترجمة الموجهة.

و في هذه عملية الترجمة، استعمال

الترجمة باللسان لأن المترجم تحاول اللغة و

القول مباشرة. "أما استعمال الترجمة

باللسان فيجوز المترجم أن يتحول اللغة و

القول مباشرة و سريعا و سديدا و بدون

الفرصة ليصلح عناصر الخطاء في لغة

الترجمة و قولها. أما استعمال الترجمة
3. أوجه التشابه بين طريقة ترجمة الكتب

العربية إلى اللغة الإنجليزية في مركز اللغة الإنجليزيةو طريقة ترجمة الكتب العربية إلى اللغة الإندونيسية في مركز اللغة العربية فونجاء دار السلام الإسلامي فوتوءان دايا باميكاسن

أوجه التشابه بين طريقة الترجمة إلى

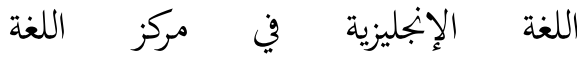

الإنجليزيةوطريقة الترجمة إلى اللغة

الإندونيسية في مركز اللغة العربية كما يلي :

عملية الترجمة في مركز اللغة

الإنجليزيةو في مركز اللغة العربية

\begin{tabular}{|c|c|}
\hline مركز اللغة العربية & مركز اللغة \\
\hline 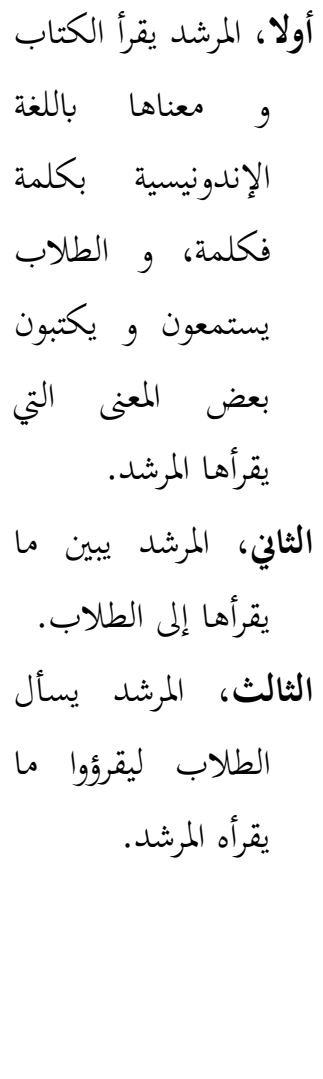 & 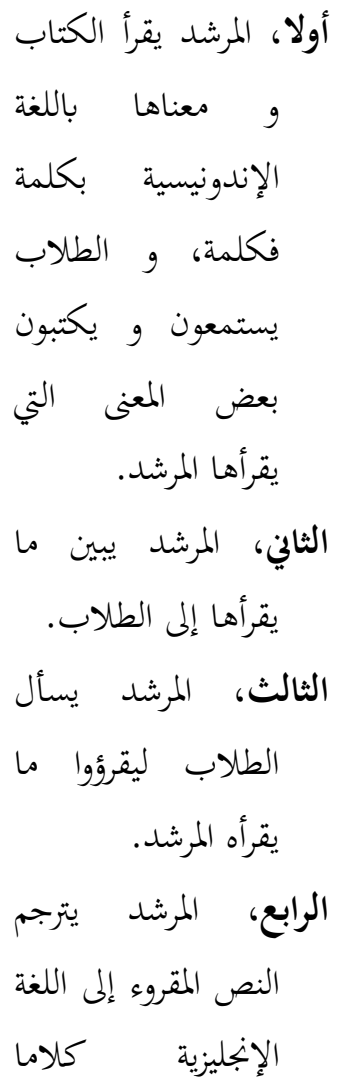 \\
\hline
\end{tabular}


أ) نتيجة الترجمة من الطلاب بمركز اللغة

$$
\text { الإنجليزية }
$$

\begin{tabular}{|c|c|}
\hline The religious low & الأحكام الشرعية \\
\hline The first low & الحهم الأول \\
\hline $\begin{array}{l}\text { Is hijab obligated for } \\
\text { all of womens? }\end{array}$ & هل يلى بجيع النساء الحجاب \\
\hline $\begin{array}{l}\text { The verse } A l- \\
\text { Karimah indicate }\end{array}$ & يدل ظاهر الأية الكريمة \\
\hline $\begin{array}{l}\text { That hijab is } \\
\text { obligated to all of } \\
\text { mukallaf moslem } \\
\text { women by syara' }\end{array}$ & مفروض أن الحجاب على مؤمنات (المكلفات \\
\hline And they are & و هن \\
\hline Muslims & المسلمات \\
\hline Independent & الحرائر \\
\hline Mature women & البالغات \\
\hline As Allah decree & لقوله تعالى \\
\hline $\begin{array}{l}\text { Oh Prophet, tell to } \\
\text { your wives and your } \\
\text { daughtersand the } \\
\text { women of believers: } \\
\text { to draw their veil all } \\
\text { of over their body }\end{array}$ & 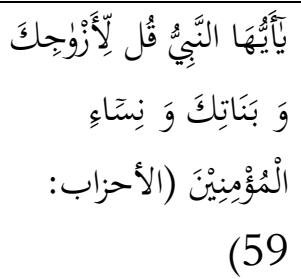 \\
\hline
\end{tabular}

ب)نتيجة الترجمة من الطلاب بمركز اللغة العربية

\begin{tabular}{|c|c|}
\hline $\begin{array}{l}\text { Adapun ini adalah } \\
\text { hukum-hukum }\end{array}$ & الأحكام \\
\hline $\begin{array}{l}\text { Yang bersifat } \\
\text { syari'ah }\end{array}$ & الشرعية \\
\hline Hukum yang pertama & الحكم الأول \\
\hline Apakah wajib & هل يجب \\
\hline Apa hijab & الحجاب \\
\hline $\begin{array}{l}\text { Atas seluruh } \\
\text { perempuan }\end{array}$ & على جميع النساء \\
\hline
\end{tabular}

بالكتابة فعند المترجم الفرصة ليصلح عناصر الخطاء في لغة الترجمة."25

قال المرشد في مركز اللغة الإنجليزية،

السيد توفيق :"عند رأيي نترجم الكتاب

بالترجمة الحرفية. لأننا نطلب تماثلا بين $26 "$ اللغة الهدف و اللغة المنبع.

قال المرشد في مركز اللغة العربية، السيد

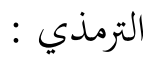

"يقال الترجمة إلى اللغة الإنجليزية بكلما فكلما آنفا.

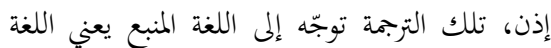

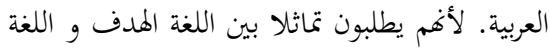

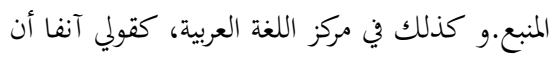

الترجمة إلى اللغة الإندونيسية في مركز اللغة العربية

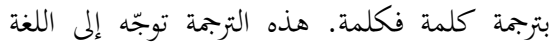

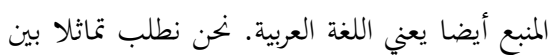

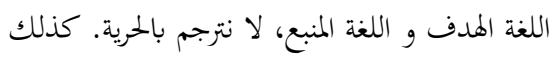

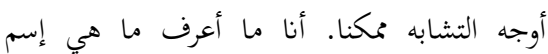

نظريته" 27

و نتيجة الترجمة من الطلاب بمركز

اللغة الإنجليزية و الطلاب بمركز اللغة

العربية عن "الأحكام الشرعية" في كتاب

تفسير آيات الأحكام : ص. 308

${ }^{25}$ Suhendra Yusuf, Teori Terjemah: Pengantar ke Arah Pendekatan Linguistik dan Sosiolinguistik. 13.

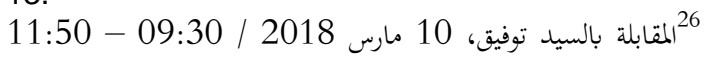
صباحا في المصلى فونجاء دار السلام.

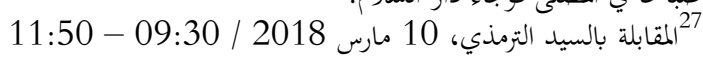
صباحا في المصلى فونجاء دار السلام. 
المساوية) : هي التي تراعى فيها محاكاة الأصل في نظمه و ترتيبيه، مثل وضع المرادف مكان مرادفه." "28

فأوجه التشابهمن ناحية المقابلةو

نتيجة الترجمة إلى اللغة الإنجليزية وإلى

اللغة الإندونيسيةو هيالترجمة الحرفيةتعنيهم

(أي المرشد في مركز اللغة الإنجليزية وفي

مركز اللغة العربية) يطلبون تماثلا بين اللغة الهدف و اللغة المنبع.

طريقة الترجمة الدلالية في الترجمة

إلى اللغة الإنجليزية بمركز اللغة الإنجليزية و

طريقة الترجمة كلمة فكلمة في الترجمة إلى

اللغة الإندونيسية بمركز اللغة العربية

توجهان إلى اللغة المنبع و هي لغة العربية.

لأن "طريقة الترجمة التي توجه إلى اللغة

المنبع أربعة و هي طريقة الترجمة كلمة

فكلمة (word for word translation)

literal ) و طريقة الترجمة الحرفية

(translation

(faithful translation)

الدلالية (semantic translation).

إذن، أوجه التشابه بين طريقة

الترجمة إلى اللغة الإنجليزية في مركز اللغة

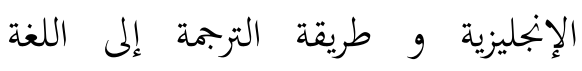

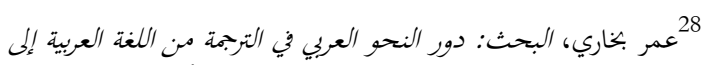

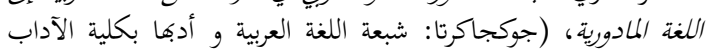

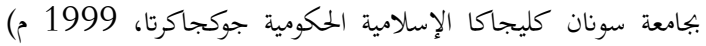
ص. 15.

${ }^{29}$ M. Zaka Al Farisi, Pedoman Penerjemahan Arab Indonesia, 53 \& 56.

\begin{tabular}{|c|c|}
\hline Menunjukkan & 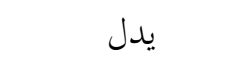 \\
\hline Apa zhahir ayat & ظاهر الأية \\
\hline Yang mulia & الكريمة - ا \\
\hline $\begin{array}{l}\text { Bahwa } \\
\text { sesungguhnya hijab }\end{array}$ & على أن الحجاب \\
\hline Adalah difardlukan & مفروض \\
\hline $\begin{array}{l}\text { Atas seluruh } \\
\text { perempuan mukmin }\end{array}$ & على جميع المؤمنات \\
\hline $\begin{array}{l}\text { Yang mukallaf- } \\
\text { mukallaf }\end{array}$ & المكلفات \\
\hline Secara syara' & شرعا \\
\hline Adapun mereka & و هن \\
\hline $\begin{array}{l}\text { Adalah perempuan- } \\
\text { perempuan muslim }\end{array}$ & المسلمات \\
\hline Yang merdeka & الحرائر \\
\hline Yang baligh & البالغات \\
\hline $\begin{array}{l}\text { Berdasarkan firman } \\
\text { Allah Ta'ala }\end{array}$ & لقوله تعالى \\
\hline Wahai Nabi & 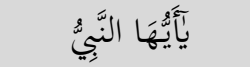 \\
\hline $\begin{array}{l}\text { Mengatakanlah siapa } \\
\text { Engkau }\end{array}$ & قُلْ \\
\hline Kepada istri-istrimu & 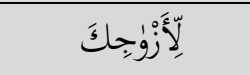 \\
\hline $\begin{array}{l}\text { Dan anak-anak } \\
\text { perempuanmu }\end{array}$ & وَ بَنَاتِكَكَ \\
\hline $\begin{array}{l}\text { Dan perempuan- } \\
\text { perempuan mukmin }\end{array}$ & وَ نَسَاءِ الْمُؤْمِنِيْنَ \\
\hline
\end{tabular}

من ناحية المقابلةو نتيجة الترجمة

إلى اللغة الإنجليزية وإلى اللغة الإندونيسية

أوجه التشابه و هي هم (أي المرشد في

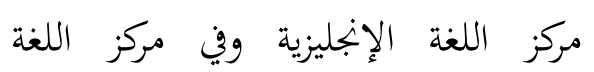

العربية) يطلبون تماثلا بين اللغة الهدف و وتركريك

$$
\text { اللغة المنبع. (المربه. }
$$

هذه الطريقة موافق بطريقة الترجمة

التي تكتب عمر بخاريفي علم بكثه

"الترجمة الحرفية (الترجمة اللفظية أو الترجمة 


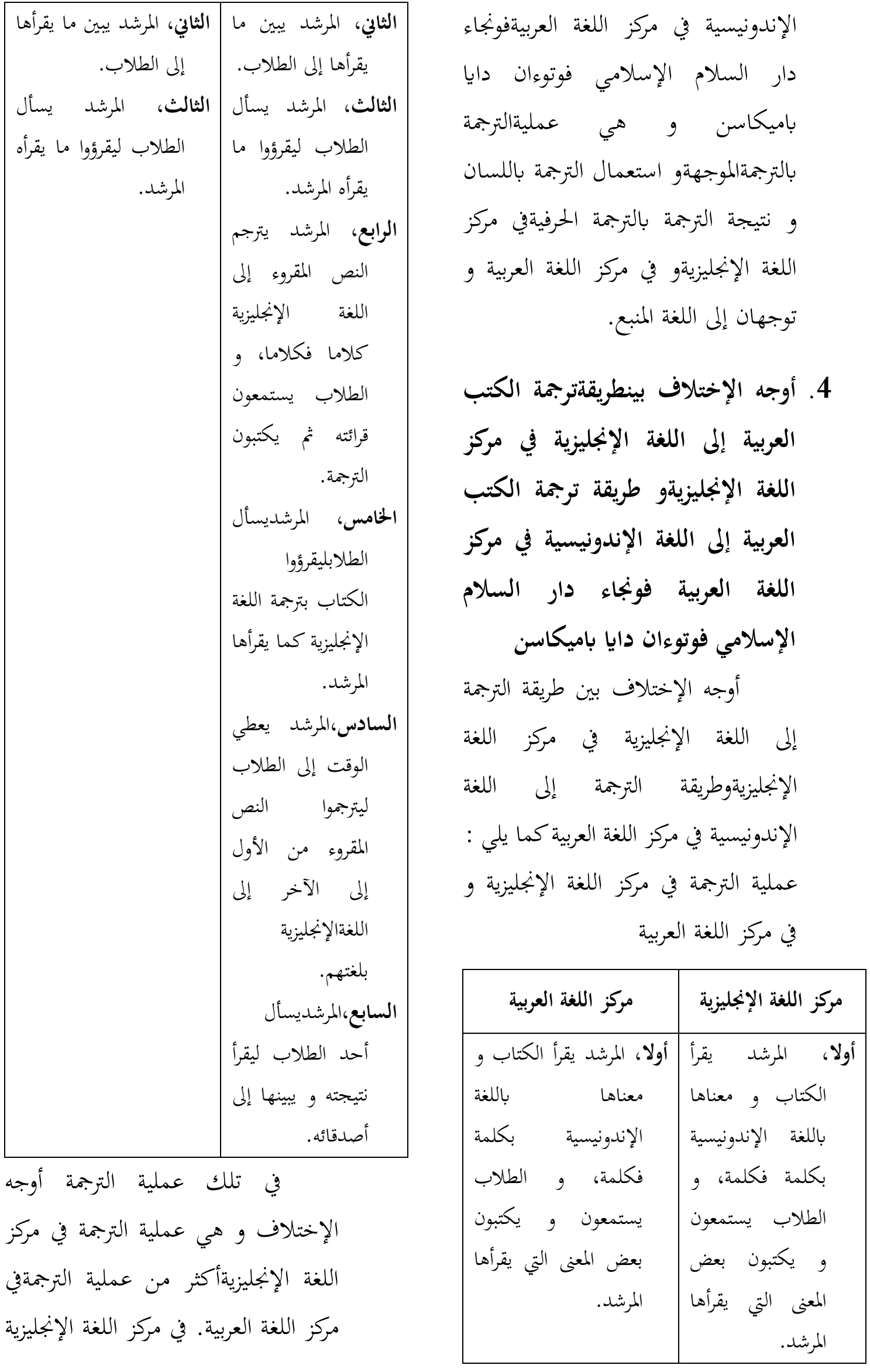




$$
\text { ب)نتيجة الترجة من الطلاب بمركز اللغة }
$$

\begin{tabular}{|c|c|}
\hline $\begin{array}{l}\text { Adapun ini adalah hukum- } \\
\text { hukum }\end{array}$ & الأحكام \\
\hline Yang bersifat syari'ah & 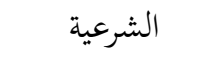 \\
\hline Hukum yang pertama & الحكم الأول \\
\hline Apakah wajib & هل يجب \\
\hline Apa hijab & الحجاب \\
\hline Atas seluruh perempuan & على جميع النساء \\
\hline Menunjukkan & يدل \\
\hline Apa zhahir ayat & ظاهر الأية \\
\hline Yang mulia & 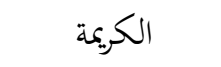 \\
\hline $\begin{array}{l}\text { Bahwa sesungguhnya } \\
\text { hijab }\end{array}$ & على أن الحجاب \\
\hline Adalah difardlukan & مفروض \\
\hline $\begin{array}{l}\text { Atas seluruh perempuan } \\
\text { mukmin }\end{array}$ & على جميع \\
\hline Yang mukallaf-mukallaf & المكلفات \\
\hline Secara syara' & شرعا \\
\hline Adapun mereka & 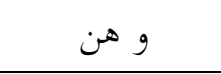 \\
\hline $\begin{array}{l}\text { Adalah perempuan- } \\
\text { perempuan muslim }\end{array}$ & المسلمات \\
\hline Yang merdeka & 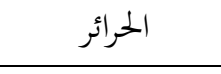 \\
\hline Yang baligh & 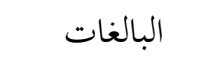 \\
\hline $\begin{array}{l}\text { Berdasarkan firman Allah } \\
\text { Ta'ala }\end{array}$ & لقوله تعالى \\
\hline Wahai Nabi & يَآيَيُهُها النَّبيُُ \\
\hline $\begin{array}{l}\text { Mengatakanlah siapa } \\
\text { Engkau }\end{array}$ & قُقل \\
\hline Kepada istri-istrimu & 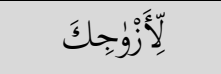 \\
\hline $\begin{array}{l}\text { Dan anak-anak } \\
\text { perempuanmu }\end{array}$ & وَ بَنَاتِكَكُ \\
\hline $\begin{array}{l}\text { Dan perempuan- } \\
\text { perempuan mukmin }\end{array}$ & وَ نِسَّاءٍ الْمُوِْْنِيْنَ \\
\hline
\end{tabular}

طريقتان ترجمة و هي طريقة الترجمة

الدلالية و طريقة الترجمة الحرية. و في تري

مركز اللغة العربية طريقة الترجمة فقط و

هي طريقة الترجمة كلمة فكلمة.

و نتيجة الترجمة من الطلاب بمركز

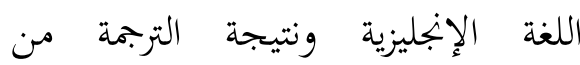

الطلاب بمركز اللغة العربية عن "الأحكام

الشرعية" قليلا في كتاب تفسير آيات

$$
\text { الأحكام : ص. } 308
$$

\begin{tabular}{|c|c|}
\hline The religious low & الأحكام الشرعية \\
\hline The first low & الحكم الأول \\
\hline $\begin{array}{l}\text { Is hijab obligated } \\
\text { for all of womens? }\end{array}$ & هل يجيع النساء الحجاب على \\
\hline $\begin{array}{l}\text { The verse Al- } \\
\text { Karimah indicate }\end{array}$ & يدل ظاهر الأية الكريمة \\
\hline $\begin{array}{l}\text { That hijab is } \\
\text { obligated to all of } \\
\text { mukallaf moslem } \\
\text { women by syara' }\end{array}$ & على أنى المجلى المكاب مفروض \\
\hline And they are & و هن \\
\hline Muslims & المسلمات \\
\hline Independent & الحرائر \\
\hline Mature women & البالغات \\
\hline As Allah decree & لقوله تعالى \\
\hline $\begin{array}{l}\text { Oh Prophet, tell to } \\
\text { your wives and } \\
\text { your } \\
\text { daughtersand the } \\
\text { women of } \\
\text { believers: to draw } \\
\text { their veil all of } \\
\text { over their body }\end{array}$ & 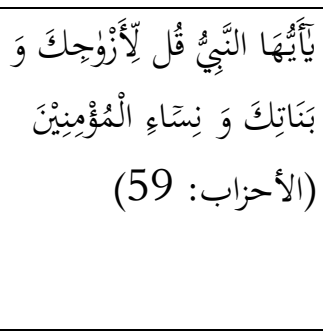 \\
\hline
\end{tabular}

أ) نتيجة الترجمة من الطلاب بمركز اللغة

$$
\text { الإنجليزية }
$$


على نظام اللغة الإنجليزية. وأمااستعمال طريقة الترجمة كلمة فكلمة أيوجوه علم التنظيم (النحو) في مركز اللغة العربية

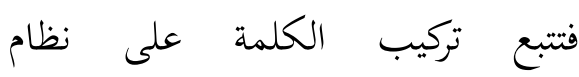
اللغةالعربية.

هذه الطريقة موافق بطريقة الترجمة

التي تكتب عمر بخاري في علم بحثه"وجوه علم التنظيم (النحو) : بحث هذا الوجه في تركيب الجملة و أجزائها و ترتيبها و تأثير كل منها بأحوال كلمة أخرى فيها و كيفية ارتباط أجزائها.و وجوه علم الدلالة : هذا الوجه بحث في معاني الكلمات و وتبدات 32 نظام الجملة الإصطلاحية.

حقيقة، في عملية الترجمة طريقة

الترجمة الدلالية و طريقة الترجمة كلمة فكلمة تكونان في عملية الترجمة المختلفة. طريقة الترجة الدلالية في عملية الترجمة الفهم و طريقة الترجمة كلمة فكلمة في

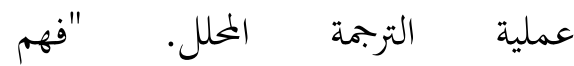
(Understanding) يفهم الملاء من المادة التي تترجه. و طراز ترجمتها و هي إنتارليغوا و الدلالية و ولته نظارية الإخبار. و و محلل (Analysis)، المترجم يجب أن يحل كل الكلام في لغة

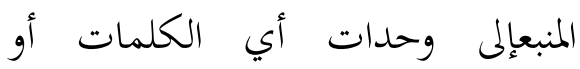

32

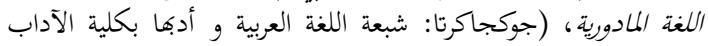
بجامعة سونان كليجاكا الإسلامية الحكومية جوكجانة الماكرتا،

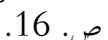

قال المرشد في مركز اللغة

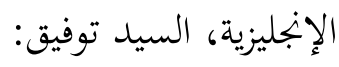

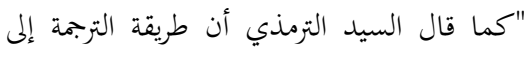
اللغة الإندونيسية و طريقة الترجمة إلى اللغة

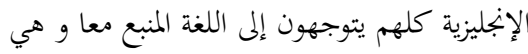
اللغة العربية. و لكن، الطريقة هذه الترجمة

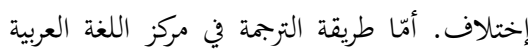

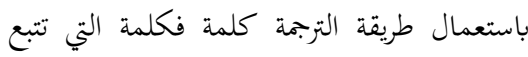
تركيب الكلمة على نظام اللغة العبية. و أمّا طريقة الترجمة في مركز اللغة الإنجليزية باستعمال

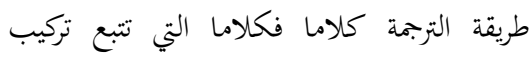
الكلمة على نظام اللغة الإنجليزية."

قالالمرشد في مركز اللغة العربية،

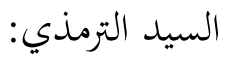

"عند رأيي أوجه الإختلاف هنا و هي أمّا الترجمة في مركز اللغة الإنجليزية استعمال طريقة الترجمة كلاما فكلاما، فالترجمة في مركز اللغة العربية

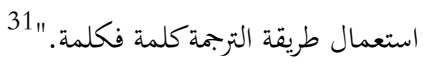

من ناحية نتيجة الترجمة من

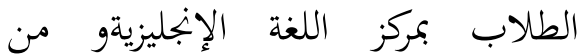
الطلاب بمركز اللغة العربية والمقابلة أوجه الإختلاف و هي استعمال طريقة الترجمة كلاما فكاما ل (أي طريقة الترجمة الدلالية) في مركز اللغة الإنجليزيةو استعمال طريقة الترجمة كلمة فكلمة في مركز اللغة العربية.أما استعمال طريقة الترجمة الدلالية(أي وجوه علم الدلالة)في مركز اللغة الإنجليزية فتتبع تركيب الكلمة

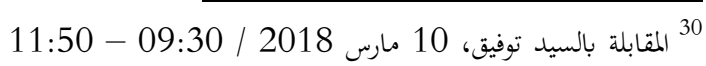
صباحا في المصلى فونجاء دار السلام.

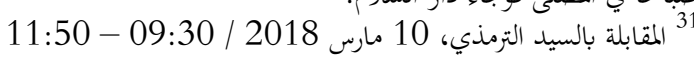
صباحا في المصلى فونجاء دار السلام. 


\section{د الإستنتاجات}

طريقة الترجمة تستعمل في الترجمة إلى اللغة

الإنجليزية في مركز اللغة الإنجليزية فونجاء دار

السلام الإسلامي فوتوءان دايا باميكاسن و هي الإنيائي فوركاء

طريقة الترجمة الدلالية بالإرشاد و طريقة الترجمة

الحرية بدون الإرشاد.و طريقة الترجمة تستعمل في الترجمة إلى اللغة الإندونيسية في مركز اللغة العربية

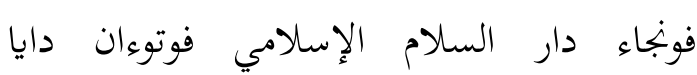
باميكاسن و هي طريقة الترجمة كلمة فكلمة. و أوجه التشابه بين طريقة الترجمة إلى اللغة

الإنجليزية في مركز اللغة الإنجليزية و طريقة الترجمة إلى اللغة الإندونيسية في مركز اللغة العربية فونجاء دار السلام الإسلامي فوتوءان دايا باميكاسن و هي: عملية الترجمة بالترجمة الموجهة و استعمال الترجمة باللسان و نتيجة الترجمة بالترجمة الحرفية و توجهان إلى اللغة المنبع.

و أوجه الإختلاف بين طريقة الترجمة إلى الى

اللغة الإنجليزية في مركز اللغة الإنجليزية و طريقة الترجمة إلى اللغة الإندونيسية في مركز اللغة العربية

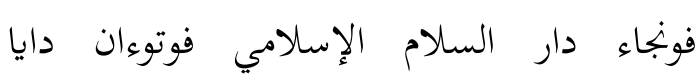
باميكاسن و هي: عملية الترجة في مركز اللغة الإنجليزية أكثر من عملية الترجمة في مركز اللغة العربية و في مركز اللغة الإنجليزية طريقتان ترجمة و هي طريقة الترجمة الدلالية و طريقة الترجمة الحرية و في مركز اللغة العربية طريقة الترجمة فقط و هي طريقة الترجمة كلمة فكلمة و استعمال طريقة الترجمة الدلالية أي وجوه علم الدلالة في مركز
العبارات. و طراز ترجمتها و هي كلمة فكلمة و النحوي و التحويل."33

إذن، أوجه الإختلاف بين طريقة الترجمة إلى اللغة الإنجليزية في مركز اللغة

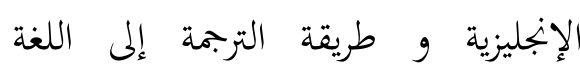
الإندونيسية في مركز اللغة العربية فونجاء دار السلام الإسلامي فوتوءان دايا باميكاسن و هي عملية الترجمة في مركز اللغة الإنجليزية أكثر من عملية الترجمة في مركز اللغة العربية. و في مركز اللغة الإنجليزية طريقتان ترجمة و هي طريقة الترجمة الدلالية و طريقة الترجمة الحرية و وهيرية في مركز اللغة العربية طريقة الترجمة فقط و هي طريقة الترجمة كلمة فكلمة. و و استعمال طريقة الترجمة الدلالية أي وجوه علم الدلالة في مركز اللغة الإنجليزية و استعمال طريقة الترجمة كلمة فكلمة أي الي لهرئه وجوه علم التنظيم (النحو) في مركز اللغة العربية. و طريقة الترجمة الدلالية في عملية الترجمة الفهم و طريقة الترجمة كلمة فكلمة في عملية الترجمة المحلل.

${ }^{33}$ A. Widyamartaya, Seni Menerjemahkan. 15. 
عمر بخاري. البحث: دور النحو العربي في الترجمة من اللغة العربية إلى اللغة المادورية. جوكجاكرتا: شبعة اللغة العربية و أدبها بكلية الآداب بجامعة سونان كليجاكا الإسلامية الحكومية جوكجاكرتا. 1999. مُحَّة إسماعيل صيني. دليل المترجم. المملكة العربية السعودية: دار العلوم للطباعة و النشر. يوسف نور عوض.1410 هـ. علم النص و نظرية الترجمة. مكة المكرمة: دار الثقه

$$
\text { للنشر و التوزيع. }
$$

\section{قائمة المراجع والمصادر الاجنبية}

Al-Farisi, Mohammad Zaka. Pedoman Penerjemahan: Arab Indonesia. Bandung: PT Remaja Rosdakarya, 2011.

Hidayatullah, Moch. Syarif. Jembatan Kata: Seluk-Beluk Penejemahan Arab- Indonesia. Jakarta: Grasindo. 2017

Machali, Rochayah. Pedoman Bagi Penerjemah. Bandung: PT Mizan Pustaka, 2009.

Moleong, Lexy J. Penelitian Kualitatif.

Bandung: Remaja Rosdakarya, 2011.

Widyamartaya, A. Seni Menerjemahkan. Yogyakarta: Anggota IKAPI, 1989.

Yusuf, Suhendra. Teori Terjemah: Pengantar ke Arah Pendekatan Linguistik dan Sosiolinguistik. Bandung: CV Mandar Maju, 1994.
اللغة الإنجليزية و استعمال طريقة الترجمة كلمة فكلمة أي وجوه علم التنظيم (النحو) في مركز اللغة العربية و طريقة الترجة الدلالية في عملية الترجمة الفهم بمركز اللغة الإنجليزية و طريقة الترجمة كلمة فكلمة في عملية الترجمة المحلل بمركز اللغة

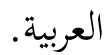

\section{References}

$$
\text { قائمة المراجع والمصادر العربية }
$$

دكتور احماده إبراهيم. الإتجاهات المعاصرة: في

$$
\text { تلدريس اللغة العربية و اللغات الحية الأخرى }
$$

لغير الناطقين كجا. القاهرة: دار الفكر

$$
\text { العربي. }
$$

ذوقان عبيدات و أصحابه. لبحث العلمي مغهوم أدوات أساليب. دار أسامة للنشرة و و

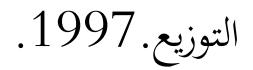

سامي عريفح و خالد حسينمصلح. في مناهج البحث العلمي و و أسالبه. عمان

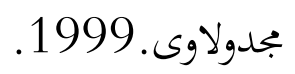
صفاء خلوصي. فن الترجمة: في ضوء الدراسات المقارنة. دار الرشيد للنشر. 1982. عبد الباقي الصافي. نظرية لغوية للترجمة. البصرة : دار الكتب. 1982.

$$
\begin{aligned}
& \text { عبد الرحمن أحمد عثمان. مناهج البحث العلمي و } \\
& \text { طريقة كتابة الرسالة الجامعية. دار جامعة }
\end{aligned}
$$

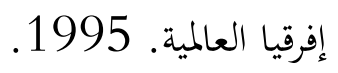

